\title{
AI for Modelling the Laser Milling of Copper Components
}

\author{
Andrés Bustillo ${ }^{1}$, Javier Sedano ${ }^{2}$, José Ramón Villar ${ }^{3}$, Leticia Curiel ${ }^{1}$, \\ and Emilio Corchado ${ }^{1}$ \\ ${ }^{1}$ Department of Civil Engineering, University of Burgos, Burgos, Spain \\ ${ }^{2}$ Department of Electromechanical Engineering. University of Burgos, Burgos, Spain \\ ${ }^{3}$ Department of Computer Science, University of Oviedo, Spain \\ abustillo@ubu.es, jsedano@ubu.es, escorchado@ubu.es, \\ lcuriel@ubu.es, villarjose@uniovi.es
}

\begin{abstract}
Laser milling is a relatively new micromanufacturing technique in the production of copper and other metallic components. This study presents multidisciplinary research, which is based on unsupervised connectionist architectures in conjunction with modelling systems, on the determination of the optimal operating conditions in this industrial process. Sensors on a laser milling centre relay the data used in this industrial case study of a machine-tool that manufactures copper components for high value micro-coolers. The two-phase application of the connectionist architectures is capable of identifying a model for the laser-milling process based on low-order models such as Black Box. The final system is capable of approximating the optimal form of the model. Finally, it is shown that the Box-Jenkins algorithm, which calculates the function of a linear system from its input and output samples, is the most appropriate model to control these industrial tasks.
\end{abstract}

\section{Introduction}

Laser milling of cooper is a complicated process due to the high conductivity and high reflectivity of this metal. Laser milling, in general, consists of the controlled evaporation of waste material due to its interaction with high-energy pulsed laser beams. The operator of a conventional milling machine is aware at all times of the amount of waste material removed, but the same can not be said of a laser milling machine. A model that could predict the exact amount of material that each laser pulse is able to remove would contribute to the industrial use and development of this new technology. The one proposed in this paper is able to optimize the manufacturing process and to control laser milling to a level of accuracy that is required for the manufacture of micro-coolers. It has been developed using a combination of conventional and Artificial Intelligence (AI) models and is applied here to data taken from micromanufacturing laser milling of copper components.

Unsupervised neural networks can be used as a preliminary phase or step before a model is established. They are used to analyze the internal structure of the data sets in order to establish that they are sufficiently informative.

The rest of the paper is organized as follows. Following the introduction, a twophase process is described to identify the optimal conditions for the industrial laser 
milling of copper components. The case study is then presented that outlines the practical application of the model. Finally, some of the different modelling systems are applied and compared, in order to select the best model in this case, before ending with a short conclusion that summarises the salient points of this work.

\section{Modelling the Laser Milling of Copper Components}

\subsection{A First Phase Using Connectionist Models}

Cooperative Maximum-Likelihood Hebbian Learning (CMLHL) [2] is applied in this study in order to analyse the internal structure of the data set under study and to establish whether it is "sufficiently informative". In the worse case, the experiments have to be performed again.

CMLHL is a Exploratory Projection Pursuit (EPP) method [1] [3], [4]. In general, EPP provides a linear projection of a data set, but it projects the data onto a set of basic vectors which help reveal the most interesting data structures; interestingness is usually defined in terms of how far removed the distribution is from the Gaussian distribution [5].

One connectionist implementation is Maximum-Likelihood Hebbian Learning (MLHL) [4], [6]. It identifies interestingness by maximising the probability of the residuals under specific probability density functions that are non-Gaussian. An extended version is the CMLHL [2] model, which is based on MLHL [4],[6] but adds lateral connections [7], [2] that have been derived from the Rectified Gaussian Distribution [5].

Considering an $\mathrm{N}$-dimensional input vector $(x)$, and an M-dimensional output vector $(y)$, with $W_{i j}$ being the weight (linking input $j$ to output $i$ ), then CMLHL can be expressed [8], [9] as:

1. Feed-forward step:

$$
y_{i}=\sum_{j=1}^{\mathbf{N}} W_{i j} x_{j}, \forall i
$$

2. Lateral activation passing:

$$
y_{i}(t+1)=\left[y_{i}(t)+\tau(b-A y)\right]^{+} .
$$

3. Feedback step:

$$
e_{j}=x_{j}-\sum_{i=1}^{M} W_{i j} y_{i}, \forall j
$$

4. Weight change:

$$
\Delta W_{i j}=\eta \cdot y_{i} \cdot \operatorname{sign}\left(e_{j}\right)\left|e_{j}\right|^{p-1} .
$$

Where: $\eta$ is the learning rate, $\tau$ is the "strength" of the lateral connections, $b$ the bias parameter, $p$ a parameter related to the energy function [2], [4], [6] and $A$ a symmetric matrix used to modify the response to the data [2]. The effect of this matrix is based on the relation between the distances separating the output neurons. 


\subsection{Second Phase}

The identification criterion evaluates which of the group of candidate models is best adapted to and which best describes the data sets collected in the experiment; i.e., given a model $M\left(\theta_{*}\right)$ its prediction error may be defined by equation (5); and a good model [8] will be that which makes the best predictions, and which produces the smallest errors when compared against the observed data. In other words, for any given data group $Z^{t}$, the ideal model will calculate the prediction error $\mathcal{E}(t, \theta)$, equation (5), in such a way that for any one $\mathrm{t}=\mathrm{N}$, a particular $\hat{\theta}_{N}$ (estimated parametrical vector) is selected so that the prediction error $\varepsilon\left(t, \hat{\theta}_{N}\right)$ in $\mathrm{t}=1,2,3 \ldots \mathrm{N}$, is made as small as possible.

$$
\varepsilon\left(t, \theta_{*}\right)=y(t)-\hat{y}\left(t \mid \theta_{*}\right) .
$$

The estimated parametrical vector $\hat{\theta}$ that minimizes the error, equation (8), is obtained from the minimization of the error function (6). This is obtained by applying the least-squares criterion for the linear regression, i.e., by applying the quadratic norm $\ell(\varepsilon)=\frac{1}{2} \varepsilon^{2}$, equation $(7)$.

$$
\begin{gathered}
V_{N}\left(\theta, Z^{N}\right)=\frac{1}{N} \sum_{t=1}^{N} \ell\left(\varepsilon_{F}(t, \theta)\right) . \\
V_{N}\left(\theta, Z^{N}\right)=\frac{1}{N} \sum_{t=1}^{N} \frac{1}{2}(y(t)-\hat{y}(t \mid \theta))^{2} . \\
\hat{\theta}=\hat{\theta}_{N}\left(Z^{N}\right)=\underset{\theta \in D_{M}}{\arg \min V_{N}\left(\theta, Z^{N}\right) .}
\end{gathered}
$$

The methodology of black-box structures has the advantage of only requiring very few explicit assumptions regarding the pattern to be identified, but that in turn makes it difficult to quantify the model that is obtained. The discrete linear models may be represented through the union between a deterministic and a stochastic part, equation (9); the term e(t) (white noise signal) includes the modelling errors and is associated with a series of random variables, of mean null value and variance $\lambda$.

$$
y(t)=G\left(q^{-1}\right) u(t)+H\left(q^{-1}\right) e(t) .
$$

The structure of a black-box model depends on the way in which the noise is modelled $H\left(q^{-1}\right)$; thus, if this value is 1 , then the OE (Output Error) model is applicable; whereas, if it is different from zero a great range of models may be applicable; one of the most common being the BJ (Box Jenkins) algorithm. This structure may be represented in the form of a general model, where $B\left(q^{-1}\right)$ is a polynomial of grade $\mathrm{nb}$, which can incorporate pure delay nk in the inputs, and $A\left(q^{-1}\right), C\left(q^{-1}\right), D\left(q^{-1}\right)$ and $F\left(q^{-1}\right)$ are autoregressive polynomials ordered as na, nc, nd, nf, respectively (10). 
Likewise, it is possible to use a predictor expression, for the on-step prediction ahead of the output $\hat{y}(t \mid \theta)$ (11). In Table 1, the generalized polynomial expressions are presented, as well as those that represent the polynomials used in the case of each particular model.

\begin{tabular}{|c|c|}
\hline$A\left(q^{-1}\right) y(t)=q^{-n_{k}} \frac{B\left(q^{-1}\right)}{F\left(q^{-1}\right)} u(t)+\frac{C\left(q^{-1}\right)}{D\left(q^{-1}\right)} e(t)$ & (10) \\
\hline$\hat{y}(t \mid \theta)=\frac{D\left(q^{-1}\right) B\left(q^{-1}\right)}{C\left(q^{-1}\right) F\left(q^{-1}\right)} u(t)+\left[1-\frac{D\left(q^{-1}\right) A\left(q^{-1}\right)}{C\left(q^{-1}\right)}\right] y(t)$ & (11) \\
\hline
\end{tabular}

Table 1. Black-box model structures

\begin{tabular}{|l|l|l|}
\hline \multicolumn{1}{|c|}{ Polynomials in $(10)$} & $\begin{array}{l}\text { Polynomials } \\
\text { used in }(10)\end{array}$ & $\begin{array}{c}\text { Name of model } \\
\text { structure }\end{array}$ \\
\hline$A\left(q^{-1}\right)=1+a_{1}\left(q^{-1}\right)+a_{2}\left(q^{-2}\right)+\cdots+a_{n_{d}}\left(q^{-n_{a}}\right)$ & B & FIR \\
$B\left(q^{-1}\right)=b_{1}\left(q^{-1}\right)+b_{2}\left(q^{-2}\right)+\cdots+b_{n_{b}}\left(q^{-n_{b}}\right)$ & AB & ARX \\
$C\left(q^{-1}\right)=1+c_{1}\left(q^{-1}\right)+c_{2}\left(q^{-2}\right)+\cdots+c_{n_{c}}\left(q^{-n_{c}}\right)$ & ABC & ARMAX \\
$D\left(q^{-1}\right)=1+d_{1}\left(q^{-1}\right)+d_{2}\left(q^{-2}\right)+\cdots+d_{n_{d}}\left(q^{-n_{d}}\right)$ & AC & ARMA \\
$F\left(q^{-1}\right)=1+f_{1}\left(q^{-1}\right)+f_{2}\left(q^{-2}\right)+\cdots+f_{n_{f}}\left(q^{-n_{f}}\right)$ & BFCD & OE \\
\end{tabular}

Procedure for Modelling the Laser Milling process. The identification procedure used to arrive at a parameterized model $\mathrm{M}$, which will eventually be selected as the best from among those that modelled the laser milling characteristics on the basis of the variable measurements, is carried out in accordance with two fundamental patterns: a first pre-analytical and then an analytical stage that assists with the determination of the parameters in the identification process and the model estimation. The pre-analysis test is run to establish the identification techniques [8], [9], [10], [11], [12], [13], the selection of the model structure and its order estimation [14], [15], the identification criterion and search methods that minimize it and the specific parametrical selection for each type of model structure.

A second validation stage ensures that the selected model meets the necessary conditions for estimation and prediction. Three tests were performed to validate the model: residual analysis $\varepsilon(t, \hat{\theta}(t))$, by means of a correlation test between inputs, residuals and their combinations; final prediction error (FPE) estimate, as explained by Akaike [16]; and the graphical comparison between desired outputs and the outcome of the models through simulation one (or k) steps before.

\section{A Case Study: Laser Milling of Copper Components}

This multidisciplinary work sets out to study and identify the optimal conditions for laser milling of computer components in a micromanufacturing technique to produce 
micro-coolers that uses a commercial Nd:YAG laser with a pulse length of $10 \mu$ sto. Three parameters of the laser process can be controlled: laser power $\left(\mathrm{u}_{1}\right)$. laser milling speed $\left(\mathrm{u}_{2}\right)$ and laser pulse frequency $\left(\mathrm{u}_{3}\right)$. The laser is integrated in a laser milling centre (DMG Lasertec 40).

To simplify this industrial problem a test piece was designed and used in all of the laser milling experiments. It consisted on an inverted, truncated, pyramid profile that had to be laser milled on a flat metallic piece of copper. The truncated pyramid had angles of $135^{\circ}$, and a depth of $1 \mathrm{~mm}$, but as the optimized parameters for the laser milling of the copper were not known at that point in time, both parameters showed errors, which are referred to, in this paper, as angle error $\left(\mathrm{y}_{1}\right)$ and depth error $\left(\mathrm{y}_{2}\right)$. A third parameter to be considered was the surface roughness of the milled piece $\left(\mathrm{y}_{3}\right)$. This variable also had to be optimized, because the industrial process required a precise geometrical shape, but also a good surface roughness of the piece. We applied different modelling systems to achieve the optimal conditions of these three parameters.

Table 2. Variables, units and values used during the experiments. All values are common to this laser milling process. Output $y(t)$, Input $u(t)$.

\begin{tabular}{|c|c|}
\hline \multicolumn{1}{|c|}{ Variable (Units) } & Range \\
\hline$\circ$ Angle error of the test piece, $\mathrm{y} 1(\mathrm{t})$ & -1 to 1 \\
\hline$\circ$ Depth error of the test piece, $\mathrm{y} 2(\mathrm{t})$ & -1 to 1 \\
\hline$\circ$ Surface roughness of the test piece $(\mu \mathrm{m}), \mathrm{y} 3(\mathrm{t})$ & 0.8 to 15 \\
\hline$\circ \quad \begin{array}{l}\text { Laser power in percent of the maximum power } \\
\text { performed by the laser }(\%), \mathrm{u} 1(\mathrm{t}) .\end{array}$ & 20 to 100 \\
\hline$\circ \quad$ Laser milling speed $(\mathrm{mm} / \mathrm{s}), \mathrm{u} 2(\mathrm{t})$. & 200 to 800 \\
\hline$\circ$ Laser pulse frequency $(\mathrm{kHz}), \mathrm{u} 3(\mathrm{t})$. & 20 to 100 \\
\hline
\end{tabular}

The experimental design was performed on a Taguchi L25 with 3 input parameters and 5 levels, so as to include the entire range of laser milling settings that are controllable by the operator. Table 2 summarizes the input and output variables of the experiment. The experiment was performed on the test piece described above. After the laser milling, actual inverted pyramid depth, wall angle and surface roughness of the bottom surface were measured using optical devices. The two first measurements were compared with the nominal values in the CAD model, thereby obtaining the two errors $\left(\mathrm{y}_{1}\right.$ and $\left.\mathrm{y}_{2}\right)$. The test piece and the prototype were described in detail beforehand [17].

\subsection{Application of the Two Phases of the Modelling System}

The experiments have been organized into two phases.

- Phase 1. Initial identification of the internal structure of the data set. Application of several unsupervised neural models.

- Phase 2. Final identification of the model that best defines the dynamic of the laser milling process. 


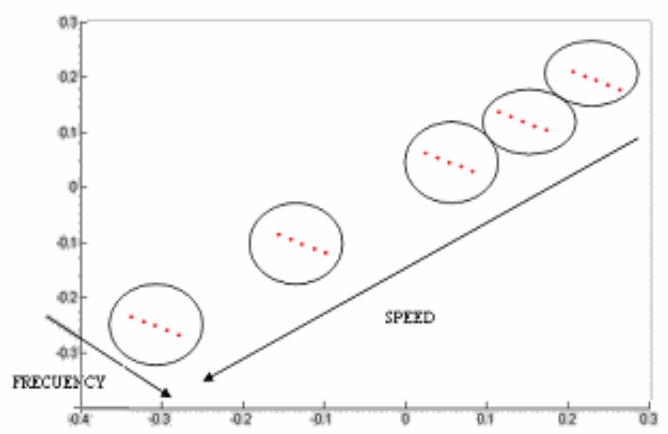

Fig. 1. The first of two projections obtained by CMLHL

Phase 1. Figure 1 shows the results obtained from the first two CMLHL projections. We can see how this method identifies a clear structure of five clusters ordered by speed and by frequency, which indicates that the data analysed is sufficiently informative.

Phase 2. Modelling the laser milling process. Figure 2, shows the results of output $\mathrm{y}_{1}(\mathrm{t})$, angle error, $\mathrm{y}_{2}(\mathrm{t})$, depth error and $\mathrm{y}_{3}(\mathrm{t})$ surface roughness, respectively, for the different models. They show the graphic representations of the results, for ARX models, in relation to the polynomial order and the delay in the inputs; various delays for all inputs and various polynomial orders [na, $\mathrm{nb}_{1}, \mathrm{nb}_{2}, \mathrm{nb}_{3}, \mathrm{nk} 1, \mathrm{nk}_{2}, \mathrm{nk}_{3}$ ] were considered to arrive at the highest degree of precision, in accordance with the structure of the models that have been used; see Table 1. In Fig. 2, the X-axis shows the number of samples used in the validation of the model, while the $\mathrm{Y}$-axis represents the range of output variables.

Table 3 shows a comparison of the qualities of estimation and prediction of the models obtained, as a function of the model, the estimation method, and the indicators, which are defined as follows:

- The percentage representation of the estimated model (expressed as so many percent "\%") in relation to the true system: the numeric value of the normalized mean error that is computed with one-step prediction (FIT1), with tenstep prediction (FIT10), or by means of simulation (FIT). Also shown are the graphical representations of true system output and both the one-step prediction $\hat{y}_{1}(t \mid m)$, the ten-step prediction $\hat{y}_{10}(t \mid m)$, and the model simulation $\hat{y}_{\infty}(t \mid m)$.

- The loss or the error function $(\mathrm{V})$ : the numeric value of the mean square error that is calculated from the estimation data set.

- The generalization error value (NSSE): the numeric value of the mean square error that is calculated from the validation data set.

- The average generalization error value (FPE): This is the numeric value of the FPE criterion that is calculated from the estimation data set. 


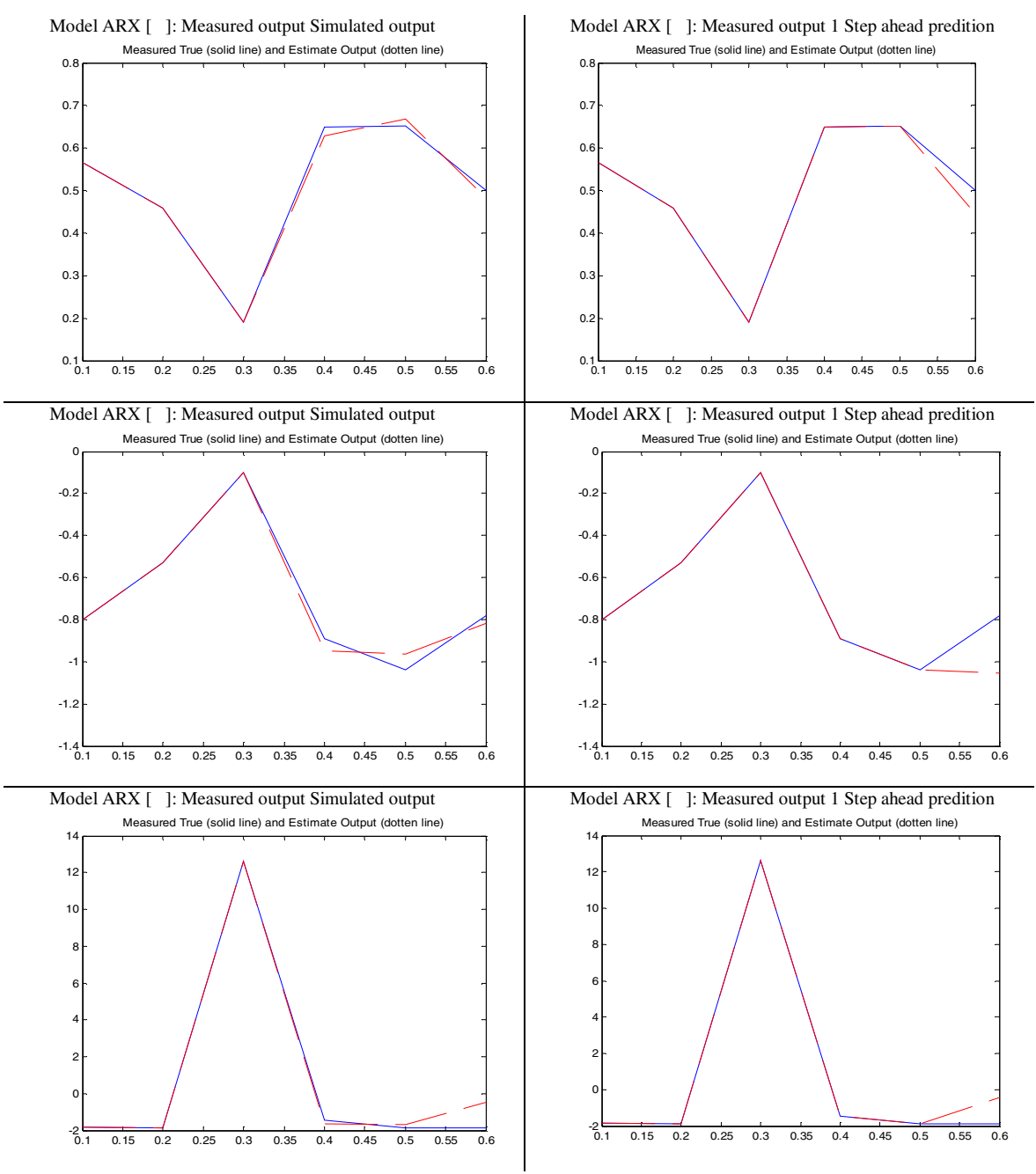

Fig. 2. Representation of measured output, simulated output and one-step-ahead prediction for three black-box models. The model generated by the ARX model for angle error, output y1(t), is shown in the upper row. On the left, measured output vs. simulated output, on the right, measured output vs. one-step-ahead prediction. The ARX model for the output y2(t), depth error, is presented in row 2 and finally, the ARX model for output $y 3(t)$, surface roughness is shown in row 3 . The validation data set was not used for the estimation of the model. The order of the structure of the model is $\left[\begin{array}{lllllll}2 & 1 & 4 & 1 & 1 & 2 & 1\end{array}\right]$ by model type. The solid line represents true measurements and the dotted line represents estimated output.

It may be seen from Fig. 2 that the ARX model is capable of simulating and predicting the error behaviour of the laser milled piece as it meet the indicators and is capable of modelling more than $90 \%$ of the true measurements. This is also evident 
Table 3. Indicator values for several proposed models

\begin{tabular}{|c|c|c|c|c|c|c|c|}
\hline \multicolumn{8}{|c|}{ Indicators and order $\left[\mathrm{na}^{2}, \mathrm{nb}_{1}, \mathrm{nb}_{2}, \mathrm{nb}_{3}, \mathrm{nk}_{1}, \mathrm{nk}_{2}, \mathrm{nk}_{3}\right]$} \\
\hline & & \multicolumn{2}{|c|}{ Angle Error } & \multicolumn{2}{|c|}{ Depth Error } & \multicolumn{2}{|c|}{ Surface roughness } \\
\hline \multicolumn{2}{|c|}{ Model } & 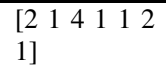 & $\begin{array}{l}{\left[\begin{array}{lllll}2 & 1 & 4 & 1 & 1 \\
3 & 1\end{array}\right]} \\
\end{array}$ & $\begin{array}{ccccc}{\left[\begin{array}{lllll}2 & 1 & 4 & 1 & 1 \\
1\end{array}\right]} & & & \end{array}$ & $\begin{array}{l}{\left[\begin{array}{llll}21 & 4 & 1 & 1 \\
3 & 1\end{array}\right]} \\
\end{array}$ & 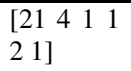 & $\begin{array}{l}{\left[\begin{array}{llll}21 & 4 & 1 & 1 \\
3 & 1\end{array}\right]} \\
\end{array}$ \\
\hline \multirow{6}{*}{$\begin{array}{l}\text { Black- } \\
\text { box } \\
\text { model, } \\
\text { ARX } \\
\text { model }\end{array}$} & $\overline{\text { FIT }}$ & $92.13 \%$ & $100 \%$ & $85.98 \%$ & $100 \%$ & $89.11 \%$ & $100 \%$ \\
\hline & FIT1 & $85.77 \%$ & $100 \%$ & $62.96 \%$ & $100 \%$ & $88.09 \%$ & $100 \%$ \\
\hline & $\overline{\text { FIT10 }}$ & $85.77 \%$ & $100 \%$ & $62.96 \%$ & $100 \%$ & $88.9 \%$ & $100 \%$ \\
\hline & $\mathrm{V}$ & 0.07 & 0.026 & 0.0197 & 0.018 & 0.249 & 0.363 \\
\hline & FPE & 0.026 & 0.069 & 0.051 & 0.047 & 0.649 & 0.088 \\
\hline & NSSE & $4.97 \exp -4$ & $\begin{array}{l}9.32 \text { exp- } \\
31\end{array}$ & 0.012 & $\begin{array}{l}3.01 \text { exp- } \\
28\end{array}$ & 0.3549 & $\begin{array}{l}2.64 \text { exp- } \\
29\end{array}$ \\
\hline
\end{tabular}

Table 4. Function and parameters that represent the behaviour for angle error of the laser milled piece

\begin{tabular}{|l|l|}
\hline \multicolumn{2}{|c|}{ Model ARX $[2141131]$} \\
\hline$A\left(q^{-1}\right) y_{1}(t)=q^{-n_{k}} B_{1}\left(q^{-1}\right) u_{1}(t)+q^{-n_{k}} B_{2}\left(q^{-1}\right) u_{2}(t)+q^{-n_{k}} B_{3}\left(q^{-1}\right) u_{3}(t)+e(t)$ \\
\hline \multicolumn{1}{|c|}{ Parameters and polynomials. } \\
\hline $\mathrm{A}(\mathrm{q})=1-1.086 \mathrm{q}^{-1}+1.195 \mathrm{q}^{-2}$ & $\mathrm{~B}_{2}(\mathrm{q})=0.003224 \mathrm{q}^{-3}+0.002786 \mathrm{q}^{-4}+$ \\
& $0.000898 \mathrm{q}^{-5}+0.004985 \mathrm{q}^{-6}$ \\
\hline $\mathrm{B}_{1}(\mathrm{q})=0.03113 \mathrm{q}^{-1}$ & $\mathrm{~B}_{3}(\mathrm{q})=0.01438 \mathrm{q}^{-1}$ \\
\hline & $\mathrm{e}(\mathrm{t})$ is white noise signal with variance \\
& 0.119 \\
\hline
\end{tabular}

Table 5. Function and parameters that represent the behaviour for the depth error of the laser milled piece

\begin{tabular}{|l|l|}
\hline \multicolumn{1}{|c|}{ Model ARX $[21411131]$} \\
\hline$A\left(q^{-1}\right) y_{1}(t)=q^{-n_{k}} B_{1}\left(q^{-1}\right) u_{1}(t)+q^{-n_{k}} B_{2}\left(q^{-1}\right) u_{2}(t)+q^{-n_{k}} B_{3}\left(q^{-1}\right) u_{3}(t)+e(t)$ \\
\hline \multicolumn{1}{|c|}{ Parameters and polynomials. } \\
\hline $\mathrm{A}(\mathrm{q})=1-2.202 \mathrm{q}^{-1}+1.653 \mathrm{q}^{-2}$ & $\mathrm{~B}_{2}(\mathrm{q})=-0.006949 \mathrm{q}^{-3}-0.005614 \mathrm{q}^{-4}-$ \\
& $0.002545 \mathrm{q}^{-5}-0.008835 \mathrm{q}^{-6}$ \\
\hline $\mathrm{B}_{1}(\mathrm{q})=-0.03203 \mathrm{q}^{-1}$ & $\mathrm{~B}_{3}(\mathrm{q})=-0.03237 \mathrm{q}^{-1}$ \\
\hline & e(t) represents white noise signal with \\
& variance 0.082 \\
\hline
\end{tabular}

from Table 3. Tables 4, 5, 6 show the function and the parameters that define the laser milling process, on the basis of the ARX model. The tests were performed using Matlab and the System Identification Toolbox. 
Table 6. Function and parameters that represent the behaviour for surface roughness of the laser milled piece

\begin{tabular}{|c|c|}
\hline \multicolumn{2}{|c|}{ Model ARX $\quad\left[\begin{array}{lllllll}2 & 1 & 4 & 1 & 1 & 3 & 1\end{array}\right]$} \\
\hline \multicolumn{2}{|c|}{$A\left(q^{-1}\right) y_{1}(t)=q^{-n_{k}} B_{1}\left(q^{-1}\right) u_{1}(t)+q^{-n_{k}} B_{2}\left(q^{-1}\right) u_{2}(t)+q^{-n_{k}} B_{3}\left(q^{-1}\right) u_{3}(t)+e(t)$} \\
\hline \multicolumn{2}{|c|}{ Parameters and polynomials. } \\
\hline $\mathrm{A}(\mathrm{q})=1+0.1501 \mathrm{q}^{-1}-0.1302 \mathrm{q}^{-2}$ & $\begin{array}{l}\mathrm{B}_{2}(\mathrm{q})=-0.004364 \mathrm{q}^{-3}-0.005079 \mathrm{q}^{-4}- \\
0.008746 \mathrm{q}^{-5}-0.005709 \mathrm{q}^{-6}\end{array}$ \\
\hline$B_{1}(q)=-0.0464 q^{-1}$ & $\mathrm{~B}_{3}(\mathrm{q})=-0.01484 \mathrm{q}^{-1}$ \\
\hline & $\begin{array}{l}\mathrm{e}(\mathrm{t}) \text { is white noise signal with variance } \\
0.153\end{array}$ \\
\hline
\end{tabular}

\section{Conclusions and Futures Lines of Work}

We have presented an investigation to study and identify the most appropriate modelling system for laser milling of copper components. Several methods were investigated to achieve the best practical solution to this interesting problem. The study shows that the BJ model is best adapted to this case, in terms of identifying the best conditions and predicting future circumstances.

It is important to emphasize that an important aspect of this research lies in the use of a two-phase model when modelling the laser milling process for copper components: a first phase, which applies projection methods to establish whether the data describing the case study is "sufficiently informative". As a consequence, the first phase eliminates one of the problems associated with these identification systems, which is that of having no prior knowledge of whether the experiment that generated the data group may be considered acceptable and will present sufficient information in order to identify the overall nature of the problem.

Future work will be focus on the study and application of other kinds of materials of industrial interest, such as steel.

\section{Acknowledgments}

This work has been made possible thanks to the support received from ASCAMM Centro Tecnológico (http://www.ascamm.es), which provided the laser milling data and performed all the laser tests. The authors would especially like to thank Mr. Pol Palouzie and Mr. Javier Diaz for their kind-spirited and useful advice. This research has been partially supported through project BU006A08 of the JCyL.

\section{References}

1. Diaconis, P., Freedman, D.: Asymptotics of Graphical Projections. The Annals of Statistics 12(3), 793-815 (1984)

2. Corchado, E., Fyfe, C.: Connectionist Techniques for the Identification and Suppression of Interfering Underlying Factors. Int. Journal of Pattern Recognition and Artificial Intelligence 17(8), 1447-1466 (2003) 
3. Friedman, J.H., Tukey, J.W.: Projection Pursuit Algorithm for Exploratory Data-Analysis. IEEE Transactions on Computers 23(9), 881-890 (1974)

4. Corchado, E., MacDonald, D., Fyfe, C.: Maximum and Minimum Likelihood Hebbian Learning for Exploratory Projection Pursuit. Data Mining and Knowledge Discovery 8(3), 203-225 (2004)

5. Seung, H.S., Socci, N.D., Lee, D.: The Rectified Gaussian Distribution. Advances in Neural Information Processing Systems 10, 350-356 (1998)

6. Fyfe, C., Corchado, E.: Maximum Likelihood Hebbian Rules. In: Proc. of the 10th European Symposium on Artificial Neural Networks (ESANN 2002), pp. 143-148 (2002)

7. Corchado, E., Han, Y., Fyfe, C.: Structuring Global Responses of Local Filters Using Lateral Connections. Journal of Experimental \& Theoretical Artificial Intelligence 15(4), 473487 (2003)

8. Ljung, L.: System Identification, Theory for the User. Prentice-Hall, Englewood Cliffs (1999)

9. Nögaard, M., Ravn, O., Poulsen, N.K., Hansen, L.K.: Neural Networks for Modelling and Control of Dynamic Systems. Springer, London (2000)

10. Söderström, T., Stoica, P.: System identification. Prentice-Hall, Englewood Cliffs (1989)

11. Nelles, O.: Nonlinear System Identification, From Classical Approaches to Neural Networks and Fuzzy Models. Springer, Heidelberg (2001)

12. Haber, R., Keviczky, L.: Nonlinear System Identification, Input-Output Modeling Approach, Part. 2: Nonlinear System structure Identification. Kluwer Academic Publishers, Dordrecht (1999)

13. Haber, R., Keviczky, L.: Nonlinear System Identification, Input-Output Modeling Approach, Part 1: Nonlinear System Parameter Estimation. Kluwer Academic Publishers, Dordrecht (1999)

14. Stoica, P., Söderström, T.: A useful parametrization for optimal experimental design. In: IEEE Trans. Automatic. Control, vol. AC-27 (1982)

15. He, X., Asada, H.: A new method for identifying orders of input-output models for nonlinear dynamic systems. In: Proc. Of the American Control Conf., S.F., California, pp. 25202523 (1993)

16. Akaike, H.: Fitting autoregressive models for prediction. Ann. Inst. Stat. Math. 20, 425439 (1969)

17. Arias, G., Ciurana, J., Planta, X., Crehuet, A.: Analyzing Process Parameters that influence laser machining of hardened steel using Taguchi method. In: Proceedings of 52nd International Technical Conference SAMPE 2007, Baltimore (2007); ISBN 978-0-938994-72-5 